\title{
Research trends in cloud, cluster and grid computing
}

\author{
Jong Hyuk Park • Laurence T. Yang • Jinjun Chen
}

Published online: 31 May 2012

(C) Springer Science+Business Media, LLC 2012

\section{Introduction}

During the last decades, many researchers in cluster and grid computing community have been devoted to developing new and efficient algorithms and architectures for cluster and grid computing. The effort has resulted in advances in new paradigms for high performance computing systems such as cluster computing and grid computing. Nevertheless, there are still many research issues need to be addressed in the arena of cluster computing and grid computing.

This special issue invites high quality contributions addressing theoretical and practical problems in various aspects of cluster computing and grid computing, including parallel algorithms and architectures, encompassing fundamental theoretical approaches, practical experimental projects, and commercial components and systems.

Six manuscripts were finally selected for this special issue after the first, second review processes. Each manuscript selected was blindly reviewed by at least three reviewers consisting of guest editors and external reviewers.

In the following, we present a brief overview on each manuscript.

J.H. Park $(\varangle)$

Department of Computer Science and Engineering,

Seoul National University of Science and Technology

(SeoulTech), 172 Gongreung 2-dong, Nowon-gu, Seoul 139-743,

Korea

e-mail: jonghyukpark2010@gmail.com

L.T. Yang

Department of Computer Science, St. Francis Xavier University, Antigonish, NS B2G 2W5, Canada

J. Chen

Information \& Communication Technologies,

Swinburne University of Technology, Melbourne, Australia

\section{Overview}

The first paper entitled "High Performance Web Server Architecture with Kernel-level Caching," by Choi et al. proposed a solution for higher performance web service by caching dynamic data using content separation between static and dynamic portions. Also, they tried to improve some drawbacks of kHTTPd implementation such as copying data redundantly, synchronous write, and processing only static data.

The second paper entitled "Performance Implications of Non-uniform VCPU-PCPU Mapping in Virtualization Environment," by Zhong et al. focused on understanding the performance implications of the non-uniform VCPU-PCPU mapping in a virtualization system. They also demonstrated that the presence of the non-uniform mapping has negative impacts on application's performance predictability.

Another paper in this special issue, entitled "A Dynamic Approach to Tolerate Soft Errors," by Xiong et al. proposed a new dynamic software-based approach, which is implemented by the technique dynamic binary instrumentation, to tolerate soft errors. Also they demonstrated that their approach can get higher reliability of dynamic software than those approaches which is implemented with static program protection method.

The fourth paper entitled "An Integrated Approach towards Aggressive State-Tracking Migration for Maximizing Performance Benefit in Distributed Computing," by YongHyuk Moon et al. presented a new state-tracking migration scheme that is integrated with aggressive reservation strategies such as immediate restart, greedy backfilling and selective preemption. The main contribution of this paper is an analysis of the effects of three techniques that can be used beyond the conventional migration schemes. 
The fifth paper entitled "A Hierarchical Parallel Discrete Event Simulation Kernel for Multicore Platform," by Wenjie Tang et al. tried to outline a simple Virtual coordinate system (VCS) for wireless sensor network that does not rely on multiple anchors. They showed that the new VCS is efficient in helping to guarantee delivery, since it assigns, to each sensor-node, a unique identity.

The final paper entitled "Real-Time Low Bitrate Multimedia Communication For Smart Spaces and Wireless Sensor Networks," by Kannan et al. developed a new effective extended hyper tangent version of fuzzy c-means with effective cluster center initialization for getting meaningful structure in noise data clustering or data analyzing in real world problems in order to overcome the said disadvantage of standard fuzzy c-means.

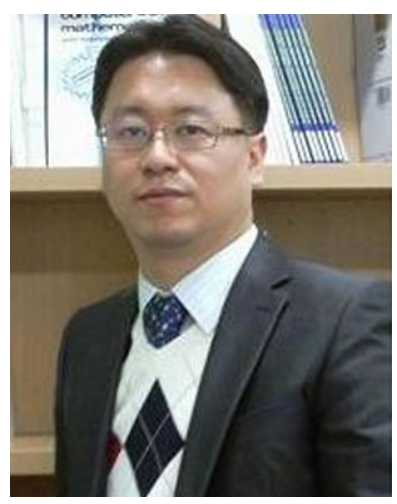

Jong Hyuk Park received his Ph.D. degree in Graduate School of Information Security from Korea University, Korea. From December, 2002 to July, 2007, Dr. Park had been a research scientist of $R \& D$ Institute, Hanwha S\&C Co., Ltd., Korea. From September, 2007 to August, 2009, He had been a professor at the Department of Computer Science and Engineering, Kyungnam University, Korea. He is now a professor at the Department of Computer Science and Engineering, Seoul National University of Science and Technology (SeoulTech), Korea. Dr. Park has published about 100 research papers in international journals and conferences. He has been serving as chairs, program committee, or organizing committee chair for many international conferences and workshops. He is a president of the Future Technology Research Association International (FTRA) and Korea Information Technology Convergence Society (KITCS). He is editor-in-chief of International Journal of Information Technology, Communications and Convergence (IJITCC), InderScience. and Journal of Convergence (JoC), FTRA Publishing. He is Associate Editor / Editor of 14 international journals including 8 journals indexed by $\mathrm{SCI}(\mathrm{E})$. In addition, he has been serving as a Guest Editor for international journals by some publishers: Springer, Elsevier, John Wiley, Oxford Univ. press, Hindawi, Emerald, Inderscience. His research interests include security and digital forensics, ubiquitous and pervasive computing, context awareness, multimedia services, etc. $\mathrm{He}$ got the best paper award in ISA- 08 conference and the outstanding leadership awards from IEEE HPCC-09 and ICA3PP-10. Dr. Park's research interests include Digital Forensics, Security, Ubiquitous and Pervasive Computing, Context Awareness, Multimedia Service, etc. He is a member of the IEEE, IEEE Computer Society, KIPS, KICS, KIISC, KMMS, KDFS and KIIT.

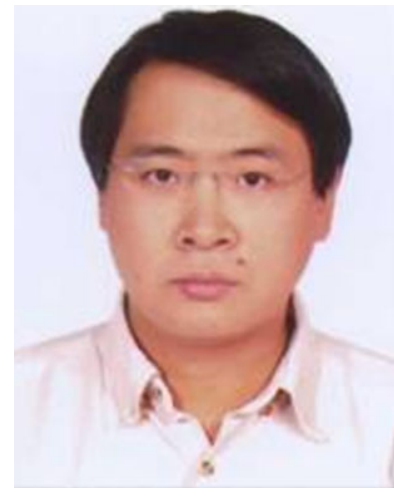

Laurence T. Yang graduated from Tsinghua University, China and got his Ph.D. in Computer Science from University of Victoria, Canada. He joined St. Francis Xavier University in 1999. His current research includes parallel and distributed computing, embedded and ubiquitous/pervasive computing. He has published many papers in various refereed journals, conference proceedings and book chapters in these areas (including around 100 international journal papers such as IEEE Journal on Selected Areas in Communications, IEEE Transactions on System, Man and Cybernetics, IEEE Transactions on Very Large Scale Integration Systems, IEEE Transactions on Industrial Informatics, IEEE Transactions on Information Technology in Biomedicine, IEEE Transactions on Parallel and Distributed Systems, IEEE Transactions on Circuit and Systems, IEEE Transactions on Service Computing, ACM Transactions on Embedded Computing Systems, IEEE Systems Journal, ACM Transactions onAutonomous and Adaptive Systems, IEEE Transactions on Vehicular Technology, ACM/Springer Journal on Mobile Networks and Applications, IEEE Intelligent Systems, Journal of Parallel and Distributed Computing, etc.). He has been involved actively in conferences and workshops as a program/general/steering conference chair (mainly as the steering co-chair of IEEE UIC/ATC, IEEE CSE, IEEE HPCC, IEEE/IFIP EUC, IEEE ISPA, IEEE PiCom, IEEE EmbeddedCom, IEEE iThings, IEEE GreenCom, etc.) and numerous conference and workshops as a program committee member. He served as the vicechair of IEEE Technical Committee of Supercomputing Applications (TCSA) until 2004, currently is the chair (elected in 2008 and 2010) of IEEE Technical Committee of Scalable Computing (TCSC), the chair of IEEE Task force on Ubiquitous Computing and Intelligence. $\mathrm{He}$ is also in the steering committee of IEEE/ACM Supercomputing conference series, and the National Resource Allocation Committee (NRAC) of Compute Canada. In addition, he is the editors-in-chief of several international journals. He is serving as an editor for many international journals. He has been acting as an author/co-author or an editor/co-editor of many books from Kluwer, Springer, Nova Science, American Scientific Publishers and John Wiley \& Sons. He has won several Best Paper Awards (including IEEE Best and Outstanding Conference Awards such as the IEEE 20th International Conference on Advanced Information Networking and Applications (IEEE AINA-06), etc); one Best Paper Nomination; Distinguished Achievement Award, 2005; Canada Foundation for Innovation Award, 2003. He has been invited to give around 20 keynote talks at various international conferences and symposia. 


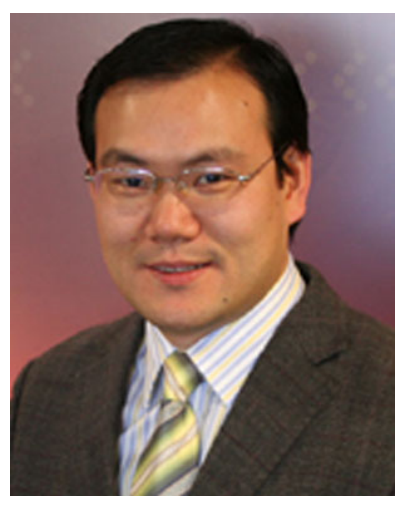

Jinjun Chen received his Ph.D. degree in Computer Science and Software Engineering from Swinburne University of Technology, Melbourne, Australia in 2007. He is currently an Associate Professor in the Faculty of Engineering and Information Technology, University of Technology, Sydney, Australia. His research interests include Scientific workflow management and applications, workflow management and applications in Web service or SOC environments, workflow management and applications in grid (service)/cloud computing environments, software verification and validation in workflow systems, QoS and resource scheduling in distributed computing systems such as cloud computing, service oriented computing, semantics and knowledge management, cloud computing. 